\title{
Mental Health Services in Rural China: A Qualitative Study of Primary Health Care Providers
}

\author{
Zhenyu Ma, ${ }^{1}$ Hui Huang, ${ }^{1}$ Qiang Chen, ${ }^{2}$ Faqin Chen, ${ }^{3}$ Abu S. Abdullah, \\ Guanghui Nie, ${ }^{1}$ Qiming Feng, ${ }^{1}$ and Bo Wei ${ }^{1}$ \\ ${ }^{1}$ School of Public Health, Guangxi Medical University, Nanning, Guangxi 530021, China \\ ${ }^{2}$ Guangxi Zhuang Autonomous Region Brain Hospital, Liuzhou, Guangxi 545005, China \\ ${ }^{3}$ Youjiang Medical University for Nationalities, Baise, Guangxi 533099, China \\ ${ }^{4}$ Boston University School of Medicine, Boston Medical Center, Boston, MA 02118, USA
}

Correspondence should be addressed to Qiming Feng; fengqm1963@hotmail.com and Bo Wei; weibogx@163.com

Received 16 August 2015; Revised 9 November 2015; Accepted 9 December 2015

Academic Editor: Abdulbari Bener

Copyright (C) 2015 Zhenyu Ma et al. This is an open access article distributed under the Creative Commons Attribution License, which permits unrestricted use, distribution, and reproduction in any medium, provided the original work is properly cited.

\begin{abstract}
This study aimed to understand the challenges that primary health care providers faced in the process of delivering mental healthcare and assess their attitudes towards patients with mental health problems. In-depth interviews were conducted among 42 primary health care providers in two counties of Guangxi province, China. All interviews were audio-recorded and analyzed thematically. Primary health care providers in both counties faced the same difficulties: lack of professional knowledge, fear of patients' attack, more extra work, and less subsidies. However, most of primary health care providers (30/42) were still willing to do mental healthcare management. All the interviewees considered that communication skills with patients and their family members, proper attitude (without discrimination), and the professional knowledge of mental health are required. There are still several participants (15/42) who showed negative attitude toward mental disorders. Nearly all the respondents (39/42) emphasized the importance of increasing their income or subsidies by the government. This qualitative study provides insights into mental health services in rural communities of Guangxi and identified issues that could be considered in engaging primary health care providers in the management of mental disorders.
\end{abstract}

\section{Introduction}

Mental health conditions affect millions of people in the world. The World Health Organization (WHO) estimates that 151 million people suffer from depression and 26 million people from schizophrenia; 125 million people are affected by alcohol use disorders [1]. In low-income countries, depression represents almost as large of a problem as does malaria (3.2\% versus $4.0 \%$ of the total disease burden) [2]. The WHO advocates that mental health services should be available in the grassroots level and integrated systematically into primary health care (PHC) [3]. Community-based services are widely regarded as the best approach for providing mental health treatment and care. At the same time primary health care providers are the basic health service resources, as well as the only resource of mental health service in rural areas. The Chinese Mental Health Work Plan (2012-2015) was outlined to equip $90 \%$ of community health service centers or conditional township health centers with full-time or parttime medical personnel who would engage in delivering care for mental health [4].

Guangxi is a less developed province in China and lacks mental health professional, especially in rural communities. At the same time, the available mental health services that are available in the city level hospital, mainly through the psychiatric departments, are not reachable by the rural population due to financial hardships, stigma, and distance [5]. Our earlier study reported a high prevalence of mental disorders $(26.81 \%)$ and less utilization of health care services or selfmedication among patients living in rural Guangxi [6]. Thus, the improvement and development of community mental health care has to rely on the primary health care providers, also known as "village doctors" who are usually from the same community and are familiar with the community members 
in the area. Primary health care providers not only are the general practitioners of primary health care but also play a mediating role among psychiatric institutions, psychiatrists, and the community population. Without this medium, psychiatrists are difficult to carry out mental health service at the grassroots level. As one of the indispensable groups in primary health care, primary health care providers' attitude towards the mental disorder patients would greatly affect the quality of health service. Evidence based guidelines for nonspecialists to enable them to identify and manage mental disorders were being carried out in low- and middle-income countries [7]. However, there were lack of understanding of the challenges that primary health care providers faced in the process of carrying out mental health work and their attitudes towards mental disorders. Thus, we conducted this qualitative study to compare the primary health care providers between two different mental health service models in rural Guangxi by conducting in-depth interviews (IDIs). The study aims to understand the challenges that primary health care providers faced in the process of carrying out mental health work and to realize their attitudes towards mental patients and so forth. This study is of great importance to guide the community mental health services in rural China.

\section{Methods}

2.1. Sample and Settings. There were two models of mental health services in rural Guangxi: one is hospital-community integrated service model and the other is the psychiatric hospital-centric model. We selected participants from the two model areas. In this study, the sample area is Liujiang County, the proportion of the population accounted for $89.7 \%$ of Zhuang ethnicity. The National Continuing Management and Intervention Program for Psychoses (686 Program) has been developed in Liujiang County since 2006 [8]. With a new hospital-community integrated service model, this program has actually covered 500000 general population in 11 towns. However, Liucheng County is the control area in this study, and its socioeconomic development level and capacity of rural health services are comparable with Liujiang County, and it still follows the traditional hospital-centric model of community mental health services.

Participants were primary health care providers engaged in community mental health services who were randomly selected from Liujiang and Liucheng Counties, respectively.

2.2. Procedures. Participants were recruited, during JuneJuly 2013, through the hospital liaisons in each township health center. Interviewers introduced themselves as being researchers from the School of Public Health of Guangxi Medical University conducting a study on primary mental health services. Researchers then explained the format of the in-depth interviews (IDIs) and provided assurance that all responses would be completely anonymous. The IDIs were conducted in a location convenient for the participants and took about 60 minutes.

2.3. Data Collection. A team of researchers with experience in conducting qualitative research collected the data. All of the IDIs were conducted in Mandarin Chinese or the local Guangxi dialect using a semistructured interview guide and audio-recorded. Open-ended questions were used in a semistructured interview approach. The guide included questions and queries on the following six themes: the situation of mental health service in PHC, main difficulties to provide mental health care, willingness to provide mental health care, required abilities of the primary health care providers, attitude toward mental disorders, and how to improve mental health care in rural area. Interviewers were graduates students at the School of Public Health of Guangxi Medical University and attended a 3-day training course on qualitative research methods and rural mental health services research. The training also included a session on the ethical aspects of human subject research. All the participants provided their written informed consent, including guidelines, relevant risks, and benefits. To collect data, interviewers recorded the session with a digital voice recorder under permission from the participants. To compensate for their time, each participant was given a cash amount of RMB 50 (US\$8). The study was approved by the Ethics Committee of the Guangxi Medical University.

2.4. Analysis. The interviewers discussed and summarized the content of each IDI and reviewed the notes taken immediately after the IDI. These debriefings were useful (i) to identify most important themes and ideas and (ii) to assess the need for any modification in the subsequent IDIs. Audio recordings of each interview were transcribed verbatim into Chinese. Content analysis was conducted following the guidelines outlined in Qualitative Interviewing [9]. Two members of the research team coded each transcript independently, with discrepancies resolved through consensus. The process of coding involved identifying key themes and marking these out on the transcripts [10]. All additional notes taken during the course of the IDIs were examined to identify various themes presented in these qualitative discussions. Frequencies were used only in a general sense such as most, some, or a few.

\section{Results}

A total of 42 IDIs were conducted among primary health care providers from Liujiang and Liucheng counties. 31 (73.8\%) of the participants were males and $11(26.1 \%)$ were females. Education varied from high school or below (52.4\%) to junior college or above $(47.6 \%)$. The average length of working time was 21 years (Table 1$)$. There was no statistical difference $(P>$ 0.05 ) between the two groups in marriage, income, gender, educational level, and working time.

The findings revealed six main themes relating to mental health services in rural communities: the situation of mental health service in PHC, main difficulties to provide mental health care, willingness to provide mental health care, required abilities of the primary health care providers, attitude toward mental disorders, and how to improve mental health care in rural area. These themes are described below supplemented by participants' statements on key themes provided in Table 2. 
TABLE 1: Demographic characteristics of in-depth interview (IDI) participants $(n=42)$.

\begin{tabular}{lcc}
\hline \multirow{2}{*}{ Characteristics } & \multicolumn{2}{c}{ In-depth interviews $(n=42)$} \\
& Liujiang $(n=21)$ & Liucheng $(n=21)$ \\
\hline Gender & 16 & 15 \\
$\quad$ Males & 5 & 6 \\
$\quad$ Females & $46.8 \pm 18.1$ & $45.4 \pm 15.9$ \\
Age (mean \pm SD) & & 15 \\
Education & 17 & 6 \\
$\quad$ High school or below & 4 & $19.9 \pm 11.0$ \\
$\quad$ Junior college or above & $21.7 \pm 13.7$ & \\
Years of work (mean \pm SD) & &
\end{tabular}

3.1. The Situation of Mental Health Service in PHC. There were different working models of mental health services in the two counties. Liujiang had implemented a new hospitalcommunity integrated service model with the support of National Continuing Management and Intervention Program for Psychoses (called "686 Program") since 2006. Most of the primary health care providers in Liujiang were trained for managing severe mental illnesses. They were more knowledgeable and familiar with the management and preventive services including community education, observation, and follow-up. However, Liucheng County was still under the traditional hospital-centric model of community mental health services. The management of severe mental illnesses in Liucheng was still relatively lagging behind.

For example, one interviewee in Liujiang said, "my main job is to follow-up the mental disorders, observe their medication compliance and their life status. If they have drug side effects or abnormal conditions, I will contact with superior hospital. As the administrator of severe mental illnesses, I play a role of bridge and tie between hopital and mental ill patients, such as telling the patients to take physical examination, and to take a follow-up visit at any time. If there is something abnormal, I need to report to the superiors in time. In addition, I also should concern about the mental ill patients, talk to them and their family members with the life and job topics, in order to ease their tensions." Another said "I had a patient. He comes to the primary health center every month. I give the same medications that the psychiatrist used to give. He has been well for five years now, with no need to return to the psychiatric hospital."

But one participant in Liucheng said, "generally, I do what the township health center arranged. To telling the truth, I am rarely active to do the work, because I have many other things to do, such as managing the diabetes and hypertensions. Another reason is that there is no attack by mental illnesses in my village. So I don't need to care about them." Another PHC provider said, "formerly, the management of the mental illnesses was almost nothing. Last year I attended a meeting that I was asked to look for the mental illnesses and fill up the health forms. But we have had no management approach yet."
3.2. The Main Difficulties to Provide Mental Health Care. PHC providers both in Liujiang and Liucheng face the same difficulties in the process of providing mental health service. They said that the lack of professional knowledge, fear of being attacked by patients, more extra work, and less subsidies made mental health care service difficult.

Some of participants (15/42) described mental health care service as a difficult task. One of them said, "I do want to help patients by providing mental health care, but what can I do for them without any basic knowledge of psychiatry."

Fear of mental disorders affected PHC providers (especially the females) in providing mental health care. A female participant said, "I am afraid of the mental disorders experience relapses. The patients could not well understand our work, sometimes they curse and shout us."

More than half of participants (28/42) considered that the subsidies were too low to reflect the job's value. "The job had no subsidies in the past two years. However, we had to do the work without subsidies." Another voice was, "it takes 50 minutes by motorcycle to the far most village. How to reimburse the visiting fare is also a problem."

3.3. Willingness to Provide Mental Health Care. Even though there were kinds of difficulties during mental health care service, most of PHC providers (30/42) were still willing to do it in both counties. They considered mental health care as their duty and responsibility. One said that "it is my responsibility to do it. When I saw some of mental illnesses could go back to farm work after taking pills, I have a sense of achievement." Other participants $(8 / 42)$ thought they have to provide mental health care by the upper-level administrative requirement. Only few respondents (4/42) showed that they were not willing to provide mental health care, like saying "I don't want to do it. Nobody will work without pay. All the work that I did has no value at all."

\subsection{Required Abilities of the Primary Health Care Providers.} All the interviewees considered that the most important factor of mental health service in rural area is communication skills with patients and their family members. Then the appropriate attitude (without discrimination) and the professional knowledge of mental health are also required. One experienced PHC provider in Liujiang said, "the ability to communicate should be skillful. Firstly you should greet him. Never talk with him about the illness immediately. Talk about other things before settling down to discuss the current illness. As you are friendly with the patients, they will answer your questions about illness. For example, I used to ask the patient whether he had some porridge or not (breakfast habit in rural Guangxi), he said yes. Then I asked what he had done just now, he said he had done the farm work. Next you could ask him about the crop of sugarcane. Make sure the patient is relaxed and happy, he will tell you his illness. At that time, you can ask him which kind of medicine he take, how often he take the medicine every day, and so forth. It's the same way to find new cases suffered mental disorders. When you communicate well with the villagers, they will tell you something new." 


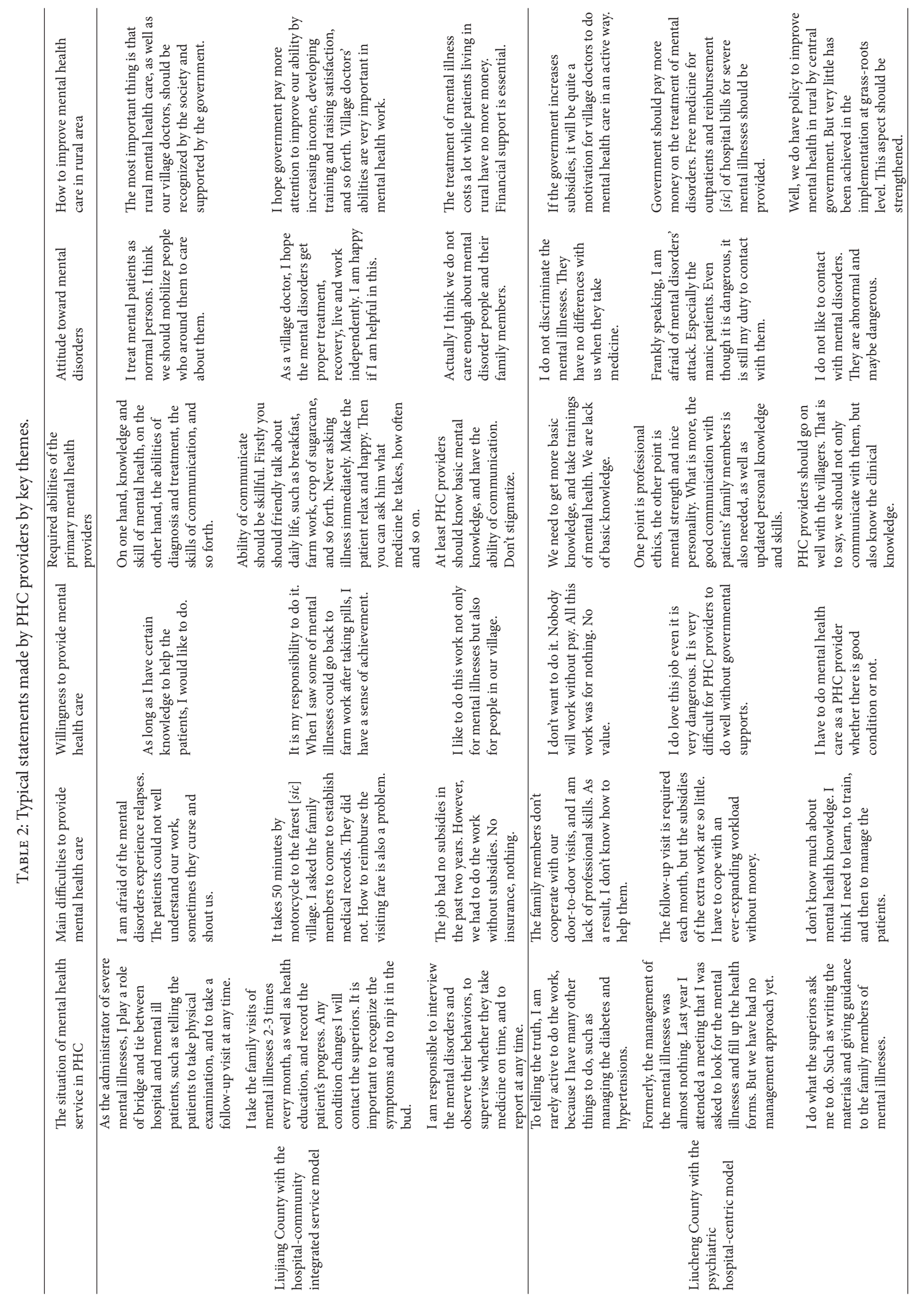


3.5. Attitude toward Mental Disorders. There are still several participants (15/42) who showed negative attitude toward mental disorders during the interview. Most of them are scared of a sudden attack by patients especially the manic and schizophrenic. One said, "most of patients are normal and follow the doctor's advice. But they still may suffer a episode of mental disorder by any irritation. It is not as safe as you provide health care to the hypertension and diabetes." Another said, "my family member worry about me to take care of mental illnesses. They worry about my safety." An unmarried female participant even said, "I am afraid of having problems with my dating, when they know that I regularly contact with mental disorders. They won't like me to do this kind of job. People usually have discrimination against those who serve the mental health patients."

\subsection{How to Improve Mental Health Care in Rural Area.} Participants provided suggestions to improve mental health care in rural area according to their practical experience. Increasing financial support was most common among the PHC providers. Every participant in Liujiang hoped that the "686 Program" would continue by with sustainable financial support. Nearly all the respondents (39/42) emphasizeddirectly or indirectly-the importance of increasing their income or subsidies by government, as one participant said, "if the government increased subsidies, it would be quite a motivation for village doctors to do mental health care in an active way."

\section{Discussion}

PHC providers engaged in community mental health services are vital to increase the availability of mental health interventions across the rural population. This quantitative research helps us to better understand health workers' attitude and notions about providing primary mental health care. The results show that there is different working status of mental health services in two counties. The PHC providers in Liujiang County with 686 Program do much more mental health interventions, including psychosocial strategies, care management, pharmacological strategies, and reducing severity of symptoms, than that of Liucheng County. The National Continuing Management and Intervention Program for Psychoses (known as the "686 Program") has provided the standardized trainings for village doctors in Liujiang to make them participate in the prevention and control network of the mental patients, such as involvement in mental disease management, mental health education, and social treatment and prevention of mental illness [11]. As a result, mental health service in Liujiang was better. The 686 program drives and motives the community mental health work. National Continuing Management and Intervention Program for Psychoses plays an important role in the development of rural community mental health service. Collins et al. also pointed that intergrating care for mental disorders into chronic disease care could reinform health services and reduce cost [12].
Lack of professional knowledge, fear of patients' attack, more extra work, and less subsidies were obstacles in providing mental health service. However, most of PHC providers were still willing to provide primary mental health care due to their professional responsibility. During the last two years, research on other 686 program pilot areas indicated that it should develop official in-service training on mental health by means of this program platform to improve the PHC providers' mental health knowledge and the ability of recognizing early symptoms among common mental disorders [13]. A recent World Health Organization and World Organization of Family Doctors report also identified that countries should improve health workers' knowledge, skills, and confidence to provide mental health interventions [14]. The respondents in our interview also pointed out that skills and knowledge of mental health were required as case managers. Improving their correct mental health cognition not only can reduce the mental patients' fear, segregation, and discrimination, but also can reduce severity of symptoms and improve daily functioning in work, social, and community life by effective mental health care management.

The findings show that some PHC providers had negative attitude toward mental disorders. Health care workers are the main workforce of providing professional health care, and their attitude towards the mental disorders is the key factor that influences the patients' recovery and promotes their social functional recovery. Health workers' attitudes affect the way of treating the patients, while psychiatric workers' attitudes are influenced by patients' symptoms [15, 16]. Inappropriate attitude towards mental disorders and their family members is a common and significant social problem. The primary mental health care providers should pay attention to this and should correct people's inappropriate attitudes. Although they provide mental health services for the patients, the providers' negative attitude towards the mental disease not only affects the way of working, such as passive working attitude, little patience, and reluctance, but also affects the patient's self-assessment and treatment compliance, reducing the result of treatment by the providers.

Nowadays, with the rapid development in science, technology, and civilization, many people including some PHC providers still have pessimistic and negative attitudes towards mental disorders. That is to say, it is very urgent task to popularize correct mental health knowledge and establish the mental health laws and regulations. PHC providers play an important role in connecting the villagers with health care institute. Government should increase the primary mental health care workers' income and welfare to eliminate unsatisfied factors existing in the work; at the same time, the appropriate incentives should be made, which provides trainings and reeducation opportunities and development space for the PHC providers, in order to improve their work enthusiasm.

Strengths of our study were not only the diverse range of respondents in terms of age, gender, education, and working years, but also the location (two counties with different mental health care model) to gather varying views. Also one limitation was that all participants were recruited from the less 
developed area Guangxi province, limiting the generalizability of the findings to attendees in other developed areas of China. However, this is the first quantitative study focusing on the thoughts and attitudes among primary mental health care providers in rural China. The findings should encourage more research in this area to promote primary health care based mental health care in rural China.

\section{Disclosure}

The authors have no financial relationships relevant to this paper to disclose. Zhenyu Ma and Hui Huang are co-first author.

\section{Conflict of Interests}

The authors have no conflict of interests relevant to this paper to disclose.

\section{Acknowledgments}

This study was supported by Grants from the China Medical Board no. 10-033 and Guangxi Natural Science Foundation no. 2012GXNSFAA053129.

\section{References}

[1] World Health Organization, Disease and Injury Regional Estimates for 2004, 2009, http://www.who.int/healthinfo/global _burden_disease/estimates_regional_2004_2008/en/.

[2] C. D. Mathers and D. Loncar, "Projections of global mortality and burden of disease from 2002 to 2030," PLoS Medicine, vol. 3, no. 11, article e442, 2006.

[3] World Health Organization, Improving Health Systems and Services for Mental Health, World Health Organization, Geneva, Switzerland, 2009.

[4] Ministry of Health of the People's Republic of China, Chinese Mental Health Work Plan (2012-2015), Ministry of Health of the People's Republic of China, Beijing, China, 2012.

[5] Q. Zhang, Mental Health Services Utilization and Related Factors among Patients with Schizophrenia in Rural Communities, Central South University, Changsha, China, 2008.

[6] B. Wei, Q.-M. Feng, Q. Cheng et al., "Epidemiological survey on the mental disorders in rural in Guangxi Zhuang Autonomous Region," Modern Preventive Medicine, vol. 38, no. 10, pp. 18011805, 2011.

[7] T. Dua, C. Barbui, N. Clark et al., "Evidence-based guidelines for mental, neurological, and substance use disorders in lowand middle-income countries: summary of WHO recommendations," PLoS Medicine, vol. 8, no. 11, Article ID e1001122, 2011.

[8] B. Wei, Q. Chen, Q.-M. Feng et al., "Implementation of comprehensive prevention and control of serious mental disorders and its effect for rural population in minority area of Guangxi," Chinese General Practice, vol. 15, no. 22, article 8A, pp. 25792582, 2012.

[9] H. J. Rubin and I. S. Rubin, Qualitative Interviewing: The Art of Hearing Data, SAGE Publications, Thousand Oaks, Calif, USA, 2nd edition, 2004.

[10] V. Braun and V. Clarke, "Using thematic analysis in psychology," Qualitative Research in Psychology, vol. 3, no. 2, pp. 77-101, 2006.
[11] Q. Chen, B. Wei, H. Tang et al., "Effect analysis of mental disease prevention, treatment and rehabilitation training among rural doctors," Journal of Applied Preventive Medicine, vol. 16, no. 5, pp. 289-290, 2010.

[12] P. Y. Collins, V. Patel, S. S. Joestl et al., "Grand challenges in global mental health," Nature, vol. 475, no. 7354, pp. 27-30, 2011.

[13] X. Xue, "Study on the effect of standardized management for patients with psychosis," Modern Preventive Medicine, vol. 40, no. 19, pp. 3610-3611, 2013.

[14] World Health Organization and World Organisation of National Colleges Academies and Academic Associations of General Practitioners/Family Physicians, Integrating Mental Health into Primary Care: A Global Perspective, World Health Organization, Wonca, Geneva, Switzerland, 2008.

[15] O. Gureje, V. O. Lasebikan, O. Ephraim-Oluwanuga, B. O. Olley, and L. Kola, "Community study of knowledge of and attitude to mental illness in Nigeria," British Journal of Psychiatry, vol. 186, pp. 436-441, 2005.

[16] G. Schomerus, C. Schwahn, A. Holzinger et al., "Evolution of public attitudes about mental illness: a systematic review and meta-analysis," Acta Psychiatrica Scandinavica, vol. 125, no. 6, pp. 440-452, 2012. 


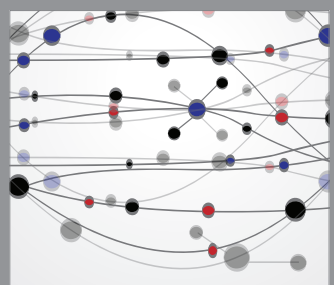

The Scientific World Journal
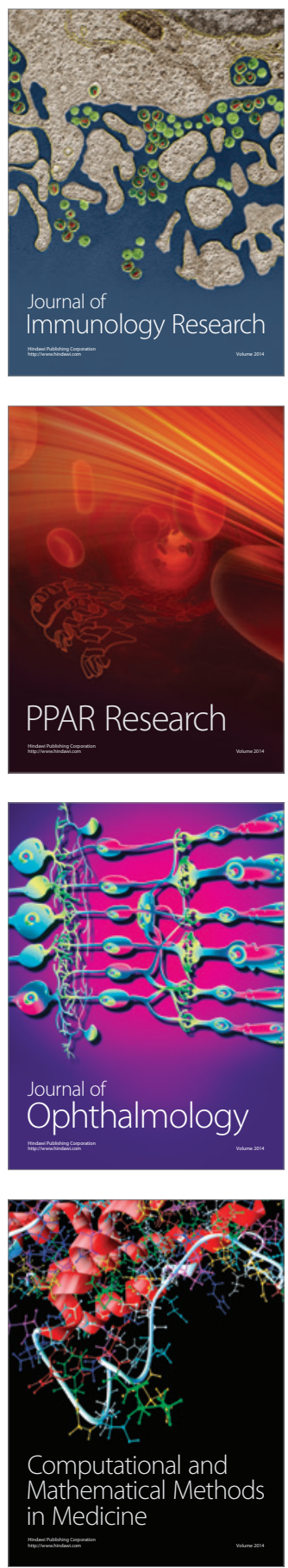

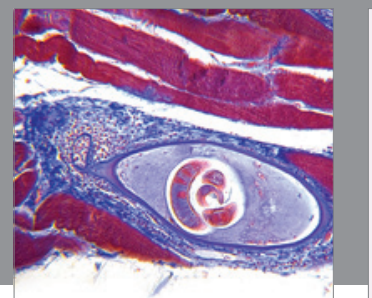

Gastroenterology

Research and Practice
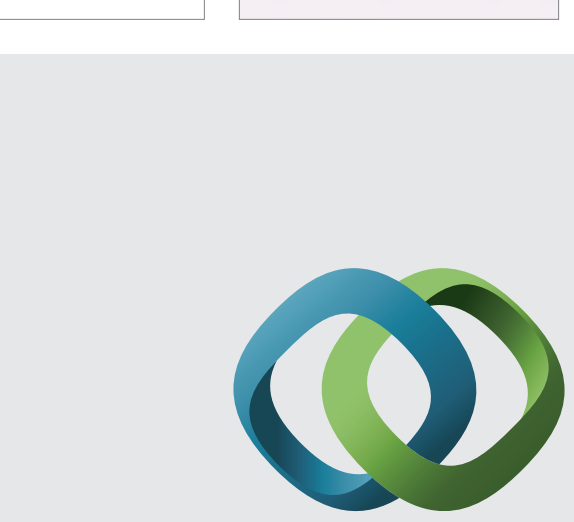

\section{Hindawi}

Submit your manuscripts at

http://www.hindawi.com
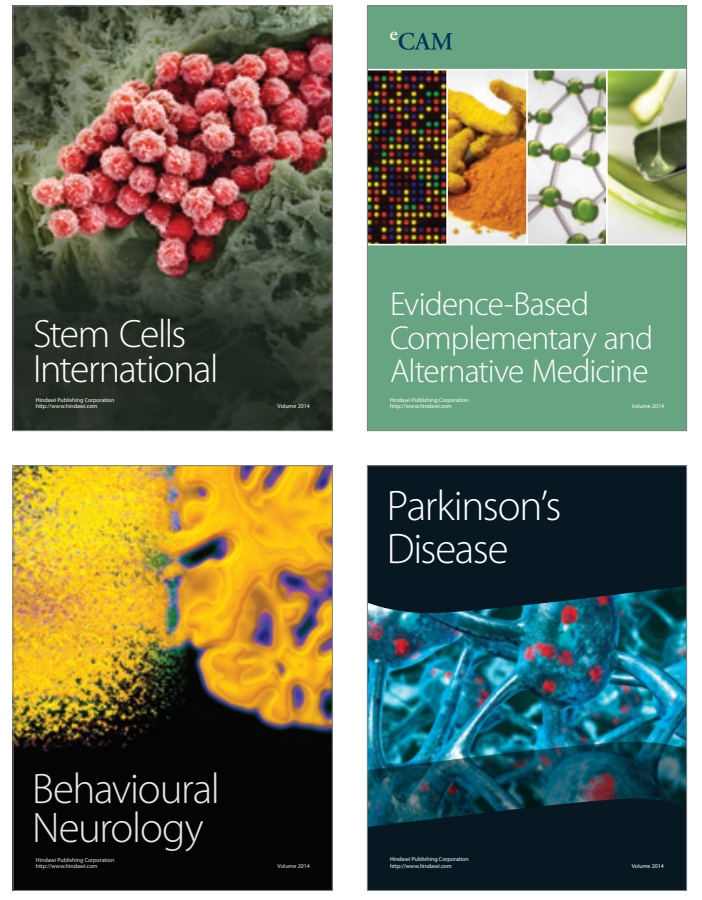
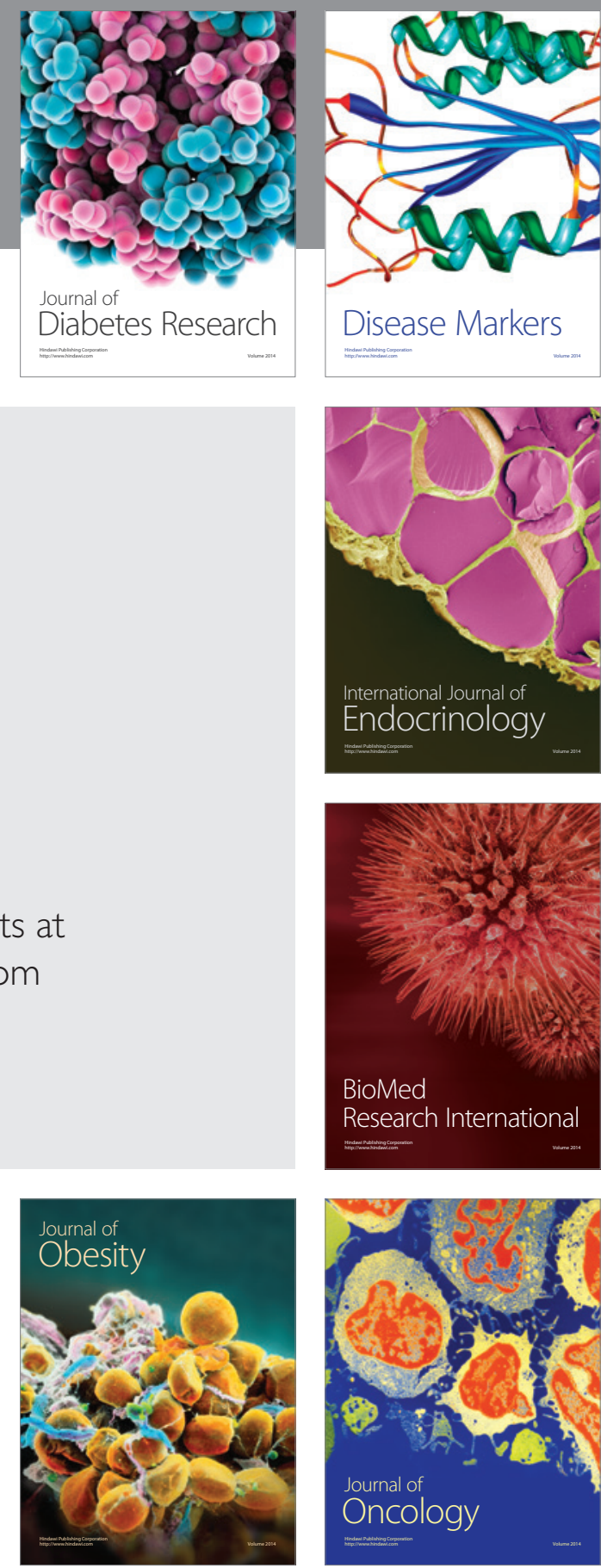

Disease Markers
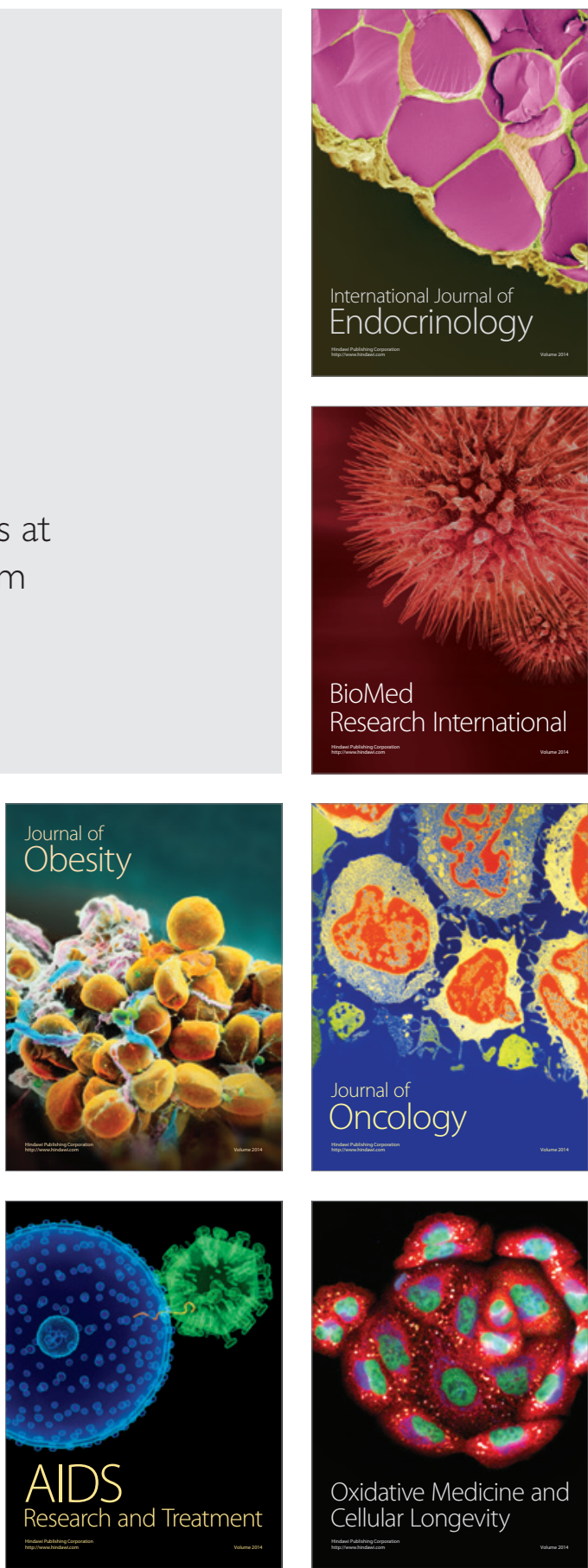